\title{
Hydrogen Isotope Fractionation in the Talc-Serpentine-Brucite-Water System: Theoretical Studies and Implications
}

\author{
Abu Asaduzzaman ${ }^{1,2}$ and Jibamitra Ganguly*,3 \\ ${ }^{1}$ School of Science, Engineering and Technology, Pennsylvania State University - Harrisburg, \\ Middletown, Pennsylvania, United States \\ ${ }^{2}$ Materials Science and Engineering and ${ }^{3}$ Department of Geosciences, University of Arizona, \\ Tucson, Arizona 85721, United States
}

\section{Supporting Information}

Table S1. Optimized lattice parameters of minerals along with corresponding experimental values.

\begin{tabular}{|l|c|c|c|c|c|c|}
\hline Parameters & \multicolumn{2}{|c|}{ Talc } & \multicolumn{2}{c|}{ Serpentine } & \multicolumn{2}{c|}{ Brucite } \\
\hline & Calc. & Exp. $^{1}$ & Calc. & Exp. $^{2}$ & Calc. & Exp. $^{3}$ \\
\hline$a$ & 9.24 & 9.29 & 5.29 & 5.34 & 3.16 & 3.15 \\
\hline$b$ & 9.10 & 9.17 & 9.22 & 9.24 & 3.16 & 3.15 \\
\hline$c$ & 9.68 & 9.46 & 14.61 & 14.68 & 4.72 & 4.77 \\
\hline$\alpha$ & 90.58 & 90.46 & 90.00 & 90.00 & 90.00 & 90.00 \\
\hline$\beta$ & 98.69 & 98.68 & 92.77 & 93.66 & 120.00 & 120.00 \\
\hline$\gamma$ & 90.03 & 90.09 & 90.00 & 90.00 & 90.00 & 90.00 \\
\hline
\end{tabular}

Table S2. Calculated vibrational frequency $\left(\mathrm{cm}^{-1}\right)$ in Talc, Serpentine and Brucite. Some of the spectroscopic frequencies are also listed for comparison. For brucite: *vibrational frequencies when all atoms in the cluster other than the $(\mathrm{OH})$ group are kept fixed in the lattice; **the three $\mathrm{Mg}$ atoms linked to an $(\mathrm{OH})$ are allowed to move

\begin{tabular}{|c|c|c|c|c|c|}
\hline Minerals & \multicolumn{2}{|c|}{ Calculated } & \multicolumn{2}{|l|}{ Measured } & \\
\hline & $\mathrm{OH}$ & OD & $\mathrm{OH}$ & OD & References \\
\hline Talc & $\begin{array}{l}3687 \\
815 \\
808 \\
402 \\
396 \\
362 \\
\end{array}$ & $\begin{array}{l}2684 \\
604 \\
596 \\
390 \\
380 \\
345 \\
\end{array}$ & $3677^{\mathrm{a}} ; 3675^{\mathrm{b}}$ & & $\begin{array}{l}{ }^{\text {a }} \text { Reference } 4 \\
\text { beference } 5\end{array}$ \\
\hline Serpentine & $\begin{array}{l}3733 \\
3729 \\
3724 \\
3704 \\
864\end{array}$ & $\begin{array}{l}2717 \\
2714 \\
2695 \\
2695 \\
650\end{array}$ & $3700^{c}$ & & ${ }^{\mathrm{C}}$ Reference 6 \\
\hline
\end{tabular}




\begin{tabular}{|c|c|c|c|c|c|}
\hline & $\begin{array}{l}855 \\
822 \\
783 \\
745 \\
702 \\
683 \\
628 \\
551 \\
516 \\
492 \\
462 \\
448 \\
431 \\
426 \\
410 \\
399 \\
380 \\
338 \\
307\end{array}$ & $\begin{array}{l}643 \\
619 \\
590 \\
584 \\
558 \\
540 \\
503 \\
495 \\
450 \\
445 \\
437 \\
421 \\
406 \\
397 \\
391 \\
376 \\
364 \\
324 \\
298\end{array}$ & & & \\
\hline Brucite* & $\begin{array}{l}3745 \\
738 \\
723 \\
353 \\
335 \\
334\end{array}$ & $\begin{array}{l}2727 \\
548 \\
541 \\
343 \\
318 \\
317\end{array}$ & $\begin{array}{l}3698^{\mathrm{d}} ; 3770^{\mathrm{e}} \\
735^{\mathrm{e}}\end{array}$ & $\begin{array}{l}2725^{\mathrm{e}} \\
545^{\mathrm{e}}\end{array}$ & 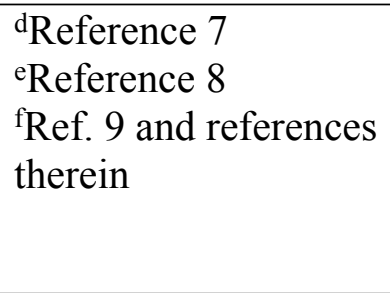 \\
\hline Brucite** & $\begin{array}{l}3746 \\
742 \\
726 \\
434 \\
403 \\
399 \\
390 \\
372 \\
352 \\
333 \\
303 \\
302 \\
271 \\
257 \\
244\end{array}$ & $\begin{array}{l}2727 \\
554 \\
546 \\
430 \\
396 \\
392 \\
387 \\
363 \\
351 \\
332 \\
302 \\
302 \\
267 \\
251 \\
238 \\
\end{array}$ & $\begin{array}{l}3698^{\mathrm{d}} ; 3770^{\mathrm{e}} \\
735^{\mathrm{e}}\end{array}$ & $\begin{array}{l}2725^{\mathrm{e}} \\
545^{\mathrm{e}}\end{array}$ & 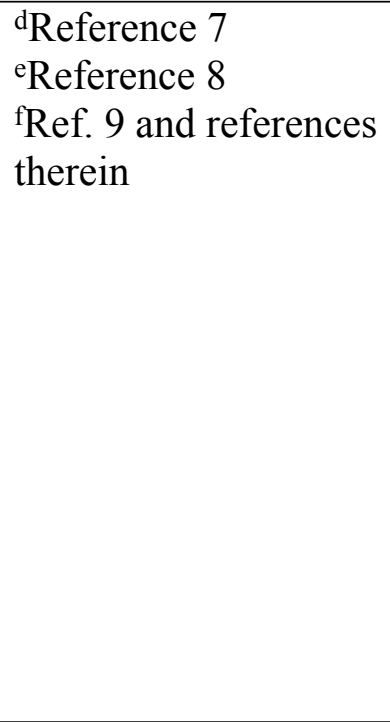 \\
\hline
\end{tabular}

\section{References}

1. Catti, M.; Ferraris, G.; Hull, S.; Pavese, A. Static Compression and H Disorder in Brucite, $\mathrm{Mg}(\mathrm{OH})_{2}$, to $11 \mathrm{GPa}$ : A Powder Neutron Diffraction Study. Phys. Chem. Miner. 1995, 22 (3). https://doi.org/10.1007/BF00202300. 
2. Falini, G.; Foresti, E.; Gazzano, M.; Gualtieri, A. F.; Leoni, M.; Lesci, I. G.; Roveri, N. TubularShaped Stoichiometric Chrysotile Nanocrystals. Chem. - Eur. J. 2004, 10 (12), 3043-3049. https://doi.org/10.1002/chem.200305685.

3. Perdikatsis, B.; Burzlaff, H. Strukturverfeinerung Am Talk Mg3[(OH)2Si4O10]. Z. Für Krist. - Cryst. Mater. 1981, 156 (3-4). https://doi.org/10.1524/zkri.1981.156.3-4.177.

4. Rosasco, G. J.; Blaha J. J. Raman Microprobe Spectra and Vibrational Mode Assignment of Talc. Appl. Spectroscopy 1980, 34, 140-144.

5. Parry, S. A.; Pawley, A. R.; Jones, R. L.; Clark, S. M. An infrared spectroscopic study of the $\mathrm{OH}$ stretching frequencies of talc and 10-Å phase to $10 \mathrm{GPa}$ American Mineralogist 2007, 92, $525-531$.

6. Kloprogge, J. T. Chapter 6 - Infrared and Raman Spectroscopies of Clay Minerals. In Development in Clay Science. Edited by W.P. Gates, J.T. Kloprogge, J. Madejová, F. Bergaya Elsevier, 2017, volume 8, pp. 150-199.

7. de Oliveira, E. F.; Hase, Y. Infrared study and isotopic effect of magnesium hydroxide. Vib. Spec. 2001, 25, 53-56.

8. Chakoumakos, B. C.; Loong, C.-K.; Schultz, A. J. Low-temperature structure and dynamics of brucite. J. Phys. Chem. B.1997, 101, 9458-9462.

9. Reynard, B; Caracas, R. D/H isotopic fractionation between brucite $\mathrm{Mg}(\mathrm{OH})_{2}$ and water from first-principles vibrational modeling. Chemical Geology 2009, 262, 159-168. 